\title{
Análisis de la satisfacción máxima del consumidor en establecimientos de alimentación: la influencia del folleto publicitario ${ }^{1}$
}

\section{Analysing the maximum level of customer satisfaction in grocery stores: the influence of feature advertising}

\author{
Maria Pilar Martínez Ruiz ${ }^{2}$ \\ Ana Isabel JimÉnez ZarCo ${ }^{3}$ \\ Alicia IzQuierdo Yusta ${ }^{4}$
}

Recibido el 25 de noviembre de 2009, aceptado el 16 de julio de 2010

$\mathrm{N}^{\mathrm{o}}$ de clasificación JEL: M31

DOI: $10.5295 / \mathrm{cdg} .100188 \mathrm{~mm}$

Reseña bibliográfica: MARTÍNEZ, M. P.; JIMÉNEZ, A. I. e IZQUIERDO, A. (2010): “Análisis de la satisfacción máxima del consumidor en establecimientos de alimentación: la influencia del folleto publicitario", Cuadernos de Gestión, Vol 10, no especial, pp. 63-83, DOI: 10.5295/cdg.100188mm

\section{Resumen:}

Partiendo de la consideración inicial de aquellos atributos del establecimiento comercial que la literatura de marketing ha identificado como fundamentales para que los distribuidores minoristas de alimentación puedan llevar a cabo sus estrategias de diferenciación, este trabajo identifica los principales factores que subyacen a dichos atributos. Todo ello, con objeto de comprender cuáles de estos factores ejercen una mayor influencia sobre el nivel más elevado de satisfacción del consumidor. Con tal fin, se ha examinado una muestra de 422 compradores que habían llevado a cabo su compra en diferentes tipos de establecimientos de alimentación en España, considerando la influencia del folleto publicitario en el comportamiento de la clientela. Este trabajo ha permitido extraer interesantes conclusiones acerca de los aspectos que más influencia presentan para que el consumidor quede totalmente satisfecho con la compra realizada en el establecimiento dependiendo de la influencia del folleto publicitario.

\footnotetext{
${ }^{1}$ Agradecimientos: Este trabajo ha sido financiado por la Fundación Ramón Areces. Acknowledgements:This work has been funded by Fundación Ramón Areces.

${ }^{2}$ Universidad de Castilla-la Mancha. Área de Comercialización e Investigación de Mercados. Facultad de Ciencias Sociales. Avenida de los Alfares, 44. Cuenca (Spain) 16071. Email:MariaPilar.Martinez@uclm.es

${ }^{3}$ Universitat Oberta de Catalunya. Estudis d'Economia i Empresa. Avinguda del Tibidabo 39-43. 08035 Barcelona (Spain). Email: ajimenezz@uoc.edu

${ }^{4}$ Universidad de Burgos. Área de Comercialización e Investigación de Mercados. Facultad de Ciencias Económicas. Plaza Infanta Elena, s/n. 09001 Burgos (Spain). Email: aliciaiz@ubu.es
} 


\title{
Palabras clave:
}

Nivel de satisfacción máximo del consumidor; establecimientos de alimentación; folleto publicitario.

\begin{abstract}
:
From the initial consideration of the store attributes that the marketing literature has identified as key in order that grocery retailers manage to design their differentiation strategies, this work identifies the main factors underlying the above mentioned attributes. The goal is to analyze which of these factors exert a bigger influence on the highest level of customer satisfaction. With this intention, we have examined a sample of 422 consumers who had carried out their purchase in different types of store formats in Spain, considering the influence of feature advertising on the clientele behavior. Interesting conclusions related to the aspects that most impact on the maximum level of customer satisfaction depending on the influence of feature advertising stem from this work.
\end{abstract}

\section{Keywords:}

Maximum customer satisfaction; grocery stores; feature advertising. 


\section{INTRODUCCIÓN}

La distribución comercial de productos de gran consumo constituye en nuestros días uno de los sectores económicos de mayor relevancia de la Unión Europea (UE, 2008). Ahora bien, en el escenario actual los operadores minoristas han de afrontar un entorno mucho más dinámico y competitivo, caracterizado por un mayor riesgo. No hay que olvidar que durante las últimas décadas se han ido produciendo numerosos procesos de fusión y adquisición de empresas que han contribuido a la configuración de un sector mucho más concentrado y con elevada participación internacional (e.g., Arnold y Fernie, 2000; Burt et al., 2002; Dupuis y Prime, 1996; Gómez, McLaughling y Wittink, 2004).

Estos cambios observados en la oferta han estado acompañados de profundas modificaciones en la demanda. A modo de ejemplo cabe mencionar, entre otros, los comportamientos y hábitos de compra actuales, que resultan muy diferenciados respecto de los que los consumidores mostraban hace tan sólo unas décadas (Berné y Martínez, 2007). Se trata de cambios que han tenido su origen: en los mayores requerimientos y formación de los consumidores (Gómez et al. 2004), en ciertas características y perfiles sociodemográficos, la proliferación de los hogares unipersonales, la mayor proporción de inmigrantes, una tasa más elevada de mujeres que trabajan fuera del hogar, la disminución paulatina del número de personas que componen los hogares o en el envejecimiento progresivo de la población (Berdié, 2007; Berné, 2006).

En esta situación, los distribuidores minoristas de productos de gran consumo, con el fin de obtener los objetivos de posicionamiento deseados, tratan de buscar la combinación adecuada de recursos y capacidades internas sobre los que construir sus estrategias competitivas (Davis-Sramek, Mentzer y Stank, 2008) basándose especialmente en la percepción de los clientes (Betancourt et al., 2007). No en vano, la práctica empresarial ha puesto de relieve cómo el conocimiento sobre el cliente supone una valiosa fuente de mejora de la capacidad competitiva de los distribuidores en el sector (e.g., Howard, 1997; Jolly y Fairhurst, 2007). Es precisamente a partir de este conocimiento cómo el responsable de tomar las decisiones comerciales puede: (a) determinar cuáles son los atributos del establecimiento y los factores subyacentes a los mismos que los clientes valoran especialmente; (b) diseñar y llevar a cabo estrategias comerciales que refuercen dichos elementos con el fin de adquirir el posicionamiento deseado y (c) satisfacer adecuadamente las necesidades de los clientes.

En esta línea de investigación, algunos estudios (e.g., Bernhardt, Donthu y Kennett, 2000; Szymanski y Henard, 2001) han evidenciado la existencia de una estrecha relación entre la percepción del consumidor de dichos atributos y factores subyacentes, y la satisfacción que obtiene en el acto de compra. Hallazgo que resulta sin duda de gran relevancia, sobre todo considerando que la satisfacción del consumidor constituye un claro antecedente de la lealtad (e.g., Yu y Dean, 2001) -lo que es clave para la posible generación y mantenimiento de vínculos duraderos con los clientes (McCollough, Berry y Yadav, 2000) - y condiciona los resultados alcanzados por las estrategias del comerciante (Betancourt et al., 2007).

Aunque la percepción que el cliente adquiere de estos atributos puede variar dependiendo de numerosos aspectos (e.g., sector de aplicación, tipo de producto, formato del establecimiento), a priori es de esperar que una percepción positiva de los mismos ejerza una 
influencia positiva en la satisfacción de compra (e.g., Gómez et al., 2004). Sin embargo, no resulta común la identificación del impacto de estos atributos y factores subyacentes sobre la satisfacción del consumidor considerando distintos aspectos clave del comportamiento del consumidor. Entre estos elementos clave es posible hablar de la respuesta promocional del consumidor a distintas actividades de promoción de ventas, que en la coyuntura económica actual, adquieren una relevancia muy significativa (Maté, 2009).

Puesto que en este escenario se ha puesto de manifiesto cómo los consumidores tienden a adquirir las mejores propuestas de valor, algunos estudiosos (e.g., Quelch, 2008) recomiendan ofrecer más promociones de precio temporales, reducir los intervalos para los descuentos en cantidad de producto, ampliar el crédito a los clientes más fieles y fijar precios más agresivos para los packs de producto más pequeños. De hecho, ante la reciente disminución de las ventas de productos de gran consumo en España, los operadores minoristas del sector están poniendo en marcha diferentes medidas para fomentar las ventas, entre las que destacan ajustes de precio a la baja -política que el grupo Mercadona ha aplicado en numerosos artículos y categorías de producto-, así como diversas actividades de promoción de ventas -básicamente descuentos de precio y regalo de unidades de producto por la compra de una o más unidades del artículo- (Maté, 2009).

Ahora bien, el éxito de dichos elementos viene condicionado no sólo por la magnitud de la rebaja que se ofrezca, sino también por la percepción que el consumidor adquiera de la misma, lo que dependerá de la adecuada o no comunicación que el distribuidor minorista realice de la promoción. Por este motivo, aunque los folletos publicitarios han sido utilizados desde hace varios años por los comerciantes minoristas en el sector de alimentación como elementos de apoyo para dar a conocer determinadas promociones a ciertos segmentos de compradores (Larson, 2004), su uso está incrementándose debido a sus características como poderosa arma competitiva en los tiempos de recesión económica actuales. No en vano, la práctica real ha mostrado cómo durante épocas de recesión, resulta crucial mantener el gasto en marketing y publicidad, lo que puede permitir mejorar la cuota de mercado a costes menores. Sobre todo, si los competidores reducen sus gastos en publicidad y los consumidores -sobre todo los más inseguros- necesitan que se reafirmen las marcas líderes (Quelch, 2008).

Teniendo en cuenta las ideas anteriores, esta investigación pretende identificar los elementos del establecimiento comercial y los factores subyacentes que la literatura relevante ha identificado como esenciales para que los operadores minoristas de productos de alimentación logren obtener un posicionamiento adecuado frente a sus competidores. Y ello, con el objetivo último de examinar la relación que existe entre la percepción del consumidor de estos elementos y su nivel máximo de satisfacción, teniendo en cuenta además la influencia del folleto publicitario.

La consideración del nivel máximo de satisfacción del consumidor resulta sin duda una de las principales aportaciones de este trabajo, teniendo en cuenta que los distribuidores minoristas con frecuencia establecen como objetivo prioritario el servir el máximo nivel de calidad percibida (Spreng y Mackoy, 1996). Además, como el estudio se realizará considerando diferentes grupos de consumidores en función de la influencia que reconocen de los folletos publicitarios a la hora de realizar la compra (por un lado, consumidores que han reconocido sentirse influidos por los folletos publicitarios a la hora de llevar a cabo sus compras de alimentación, y por otro lado, consumidores que no reconocen ningún tipo de 
influencia), se obtendrán interesantes conclusiones que dependerán del impacto del folleto publicitario.

La estructura de este trabajo es la siguiente. En primer lugar, se presentará el marco conceptual de la investigación. Seguidamente, se obtendrán los factores subyacentes a los atributos del establecimiento comercial considerados a partir de la percepción del consumidor, considerando para ello una muestra de 422 consumidores que se ha recogido en establecimientos minoristas de alimentación que operan en una ciudad española de tamaño medio (La Caixa, 2008). El análisis de esta muestra ha permitido conocer el impacto de los factores anteriormente identificados sobre la máxima satisfacción del consumidor por medio de la aplicación de un modelo de regresión logístico binario. Además, tal y cómo se comentaba con anterioridad, los resultados se han obtenido diferenciando entre consumidores en función de la influencia que el folleto publicitario representa en sus compras. De este estudio se desprenden interesantes conclusiones y recomendaciones para la gestión comercial de este tipo de establecimientos, sobre todo en relación a los atributos y factores del establecimiento comercial que los consumidores valoran en mayor medida así como su influencia sobre el nivel máximo de satisfacción del consumidor dependiendo del impacto de los folletos.

\section{MARCO CONCEPTUAL}

En la actualidad, la diferenciación del establecimiento constituye un aspecto esencial para que el minorista que opera en el sector de productos de gran consumo logre alcanzar el éxito. Si el cliente percibe que el establecimiento comercial y su oferta son superiores a los de los competidores, es posible que aumente la satisfacción que adquiere con la compra que realiza en dicho establecimiento (Gómez et al., 2004).

Resulta crucial que los responsables de acometer la gestión comercial tengan muy en cuenta esta satisfacción, ya que al constituir una respuesta afectiva a la valoración de atributos del establecimiento comercial -cuya síntesis genera la imagen del establecimiento comercial (Theodoridis y Chatzipanagiotou, 2009)-, los consumidores la utilizan para distinguir unos establecimientos de otros (e.g., Ganesh, Reynolds y Luckett, 2007; Gómez et al., 2004). Además, la satisfacción es un antecedente de las dimensiones críticas del marketing como lo son el compromiso (e.g., Johnson et al., 2001), la lealtad (e.g., Sivadas y Baker-Prewitt, 2000) o la retención de clientes (e.g., Rust y Zahorik, 1993).

Sin embargo, a pesar de la relevancia fundamental de la satisfacción, la mayoría de los trabajos realizados en esta línea de investigación no suelen analizar la influencia de la valoración del consumidor de los atributos que caracterizan al establecimiento comercial sobre la satisfacción, y más específicamente, sobre el nivel máximo de satisfacción del consumidor, que debería constituir el objetivo de cualquier operador en el sector (e.g., Hennig-Thurau y Klee, 1997; Spiller, Bolten y Kennerknecht, 2006).

Si se desea analizar la relación existente entre las percepciones del consumidor de los atributos que caracterizan al establecimiento comercial y su nivel máximo de satisfacción, se ha de identificar previamente: (1) los atributos que el cliente percibe y que resultan clave para que el distribuidor minorista logre un determinado posicionamiento en el mercado; (2) los factores que subyacen a dichos atributos considerando la importancia atribuida por 
el consumidor; y (3) la relación que existe entre la valoración de los factores por parte del consumidor y su nivel máximo de satisfacción con la compra. Con el conocimiento derivado del análisis de estos aspectos, el distribuidor minorista que opera en el sector podrá idear y llevar a cabo estrategias comerciales adaptadas a los aspectos que valoran los diversos segmentos de consumidores, lo que le otorgará mayor competitividad a largo plazo.

Al respecto, Gómez et al. (2004), Berné (2006) y Ganesh et al. (2007), entre otros, identifican diversos atributos del establecimiento comercial que, utilizados adecuadamente por los comerciantes en sus estrategias de diferenciación, son susceptibles de influir positivamente en la satisfacción de los consumidores. Entre estos atributos, cabe mencionar la atención al cliente, aspecto que resulta de gran relevancia especialmente para aquellos grupos de consumidores que disfrutan llevando a cabo procesos de socialización durante el acto de compra (e.g., Tauber, 1972). No en vano, el deseo de interactuar con otros individuos puede fomentar que algunos consumidores realicen sus compras en aquellas tiendas en las que encuentren empleados amables y comunicativos, pudiéndose considerar en estos casos que el acto de compra constituye una actividad para aliviar la sensación de soledad (Rubenstein y Shaver, 1980). Adicionalmente, Molina et al. (2006) observan cómo los beneficios de carácter social que algunos clientes buscan reflejan su necesidad de ser reconocidos como algo más que simples clientes, así como la posibilidad de tratar con alguien con el que se esté más familiarizado.

Por otra parte, los servicios adicionales del establecimiento comercial pueden influir en la satisfacción del consumidor. De esta forma, lograr que el cliente obtenga una mayor conveniencia a través de diversos servicios (e.g., horarios de apertura más amplios, parking disponible), puede aumentar su satisfacción (Berry et al., 2002). Por este motivo, no resulta extraño que los establecimientos que ofrecen los servicios buscados logren una mayor aceptación en el mercado (Malhotra, 1983), o que los consumidores prefieran realizar sus compras de textil en centros comerciales que ofrecen las combinaciones de servicios que desean (e.g., Finn y Louviere, 1990).

La atmósfera del establecimiento comercial constituye también uno de los elementos que los distribuidores minoristas consideran en sus estrategias de diferenciación al influir sobre la decisión de compra del consumidor (Kotler, 1973: 48). Sobre este particular, cabe señalar cómo algunos estudios han evidenciado la inclinación que algunos consumidores muestran a realizar sus compras en aquellos centros comerciales que les inspiran actitudes positivas (e.g., Finn y Louviere, 1990; 1996; Gentry y Burns, 1977). Por lo que en general, el tiempo que los consumidores pasan en los establecimientos y el gasto que llevan a cabo en los mismos tiende a aumentar en entornos agradables (Donovan et al., 1994). En este sentido, los compradores recreacionales que valoran especialmente el disfrute de la compra, confieren una mayor importancia a la decoración del establecimiento (Bellenger y Korgaonkar, 1980). Por este motivo, Lambert (1979) sostiene que es importante proporcionar áreas de descanso y una temperatura ambiental adecuada en los establecimientos.

La amplitud y la profundidad del surtido son valiosos elementos de la estrategia de los comerciantes minoristas en el sector, ya que permiten atender diferentes gustos y preferencias de los clientes (Dhar et al., 2001). En efecto, una mayor variedad de surtido permite generar más tráfico de clientes al establecimiento a la vez que es capaz de incentivar un mayor número de compras por cliente. Ofrecer una amplia selección de productos puede contribuir a reducir los costes percibidos en relación con los esfuerzos que se han de lle- 
var a cabo en cada acto de compra, al margen de facilitar el propio acto de compra. Por tanto, una mayor variedad del surtido puede aumentar la conveniencia que el consumidor obtiene, y por ende, su satisfacción (Dellaert et al., 1998). Comercializar marcas propias constituye también un activo de carácter estratégico para estos distribuidores puesto que contribuye a incrementar la variedad del surtido y a lograr otros objetivos relacionados con el posicionamiento a largo plazo (Binninger, 2008; Corstjens y Lal, 2000; Gomez y Rubio, 2006).

Tradicionalmente, el precio y las promociones de ventas han sido considerados como aspectos del establecimiento comercial de gran relevancia capaces de influir sobre la percepción de los consumidores. Por esta razón, los comerciantes en el sector los consideran esenciales cuando desean alcanzar un posicionamiento de precios reducidos, que suelen obtener bien mediante una política de precios reducidos o bien a través del establecimiento de descuentos de precio en categorías y productos atractivos para los consumidores (e.g., Dunne y Kahn, 1997). Aparte de los efectos beneficiosos que los descuentos de precio promocionales pueden inducir en estos establecimientos, como el incremento de las ventas de la categoría de productos en su conjunto o la creación de tráfico de clientes (Blattberg et al., 1995; Buil y Montaner, 2007; Gázquez y Sánchez, 2006; Martínez Ruiz y Mollá Descals, 2007), hay que tener en cuenta también las ventajas que se derivan de los periodos de rebajas, que pueden incentivar las compras al favorecer que el comprador identifique la posibilidad de beneficiarse de unas circunstancias especiales (Molero y Puelles, 2009).Asimismo, la calidad percibida de los productos ofrecidos es considerado otro de los atributos diferenciadores del establecimiento comercial (Binninger, 2008; Pan y Zinkhan, 2006). Sobre este particular, diversos trabajos han detectado cómo la calidad de los productos vendidos influye en el valor que el cliente percibe, resultando fundamental en la evaluación que los consumidores realizan del establecimiento (e.g., Seiders et al., 2005). La mercancía que el establecimiento ofrece ejerce una influencia sobre la reputación del comerciante, y de hecho, la calidad percibida varía en función del establecimiento en el que se efectúa la compra (Darden y Schwinghammer 1985; Pan y Zinkhan, 2006).

Finalmente, para concluir esta sección, se desea enfatizar que la valoración que los distintos segmentos de consumidores realicen de los atributos previos dependerá de aspectos tales como el tipo de producto, el formato de establecimiento comercial o la influencia de elementos diversos como los folletos publicitarios, que en la coyuntura económica actual están adquiriendo una gran relevancia (Maté, 2009), y no sólo como elementos de apoyo a otras promociones - tal y cómo se les venía considerando tradicionalmente (Larson, 2004)sino como poderosas herramientas competitivas dada su influencia sobre las percepciones de los consumidores. No hay que olvidar que los folletos publicitarios permiten simplificar los procesos de búsqueda y evaluación de alternativas por parte del consumidor (Martínez Ruiz, 2005).

No en vano, algunos trabajos (e.g., Burton et al., 1999) han puesto de manifiesto cómo la exposición a los folletos puede incrementar la probabilidad de que los consumidores no sólo visualicen ciertas propuestas de valor específicas ofrecidas por los comerciantes, sino también respondan favorablemente a las mismas. Se trata por tanto de efectos beneficiosos que serán especialmente notables si se comparan las compras de aquellos consumidores que no han sido expuestos al folleto con las de los que sí han sido expuestos (Burton et al., 1999). 


\section{DESCRIPCIÓN DE LA MUESTRA, VARIABLES Y PROPUESTA DE HIPÓTESIS}

En la sección siguiente se describirá el proceso que se ha seguido para recoger la información, las variables que se han utilizado en el estudio y la propuesta de hipótesis que se ha planteado en función de los atributos caracterizadores del establecimiento comercial y sus factores subyacentes, el nivel máximo de satisfacción del consumidor y la influencia del folleto publicitario.

\subsection{Proceso de recogida de la información y obtención de la muestra}

Mediante entrevistas personales, se administró desde el 17 de marzo al 31 de marzo de 2008 un cuestionario a compradores que habían llevado a cabo sus compras en hipermercados, supermercados, tiendas de conveniencia y establecimientos de descuento en la ciudad de Cuenca. Esta ciudad española fue escogida para realizar el estudio porque su tamaño resulta representativo del tamaño medio de la mayor parte de ciudades españolas. En torno a este periodo, algunas fuentes (e.g., El Economista, 2008; Infobae, 2008) sitúan el inicio de la recesión económica en nuestro país, lo que posteriormente provocaría el inicio de ciertos cambios en las pautas tradicionales de los consumidores españoles (e.g., El diario montañés, 2009).

Los compradores fueron contactados por los entrevistadores en sesiones de mañana y tarde a la salida de los establecimientos comerciales. El número de cuestionarios válidos recogidos ascendió a 422. El procedimiento de muestreo utilizado fue no probabilístico por conveniencia. La Tabla 1 describe la ficha técnica de la investigación.

Tabla 1

Ficha técnica del cuestionario

\begin{tabular}{l|l}
\hline Universo & $\begin{array}{l}52.980 \text { individuos residentes en la ciudad de Cuenca } \\
(\text { España } *\end{array}$ \\
\hline Unidad Muestral & Consumidores mayores de 18 años (40.075 personas) \\
\hline Ámbito geográfico & Cuenca capital \\
\hline Método de recogida de la información & Encuesta personal \\
\hline Lugar de realización de la encuesta & $\begin{array}{l}\text { Establecimientos minoristas de alimentación en régi- } \\
\text { men de libreservicio situados en el área urbana objeto } \\
\text { de consideración }\end{array}$ \\
\hline Tamaño de la muestra & 422 cuestionarios \\
\hline $\begin{array}{l}\text { Descripción de la muestra: número } \\
\text { de establecimientos y encuestas por } \\
\text { formato }\end{array}$ & $\begin{array}{l}\text { Hipermercado: } 2 ; 113 \\
\text { Supermercado: } 8 ; 245 \\
\text { Tienda de conveniencia: } 4 ; 28 \\
\text { Establecimiento de descuento: } 2 ; 26\end{array}$ \\
\hline Procedimiento de muestreo & Por conveniencia \\
\hline Fecha de trabajo de Campo & 17 al 31 de marzo de 2008 \\
\hline
\end{tabular}

*De acuerdo a los datos recogidos en La Caixa (2008). 


\subsection{Descripción de variables}

En el cuestionario se incluyeron preguntas orientadas a recoger información general sobre la experiencia de compra y la satisfacción del consumidor. Por un lado, se incorporaron preguntas relacionadas con la percepción de los consumidores sobre determinados atributos caracterizadores del establecimiento comercial. En estas variables, se solicitaba al cliente que asignara una puntuación en relación con la percepción que había obtenido respecto de diferentes atributos del establecimiento comercial en una escala de cinco puntos. Los enunciados de estas preguntas recogían los atributos más destacables identificados previamente en la literatura relevante (e.g., Frasquet y Mollá, 2000; Ganesh et al., 2007; Gómez et al., 2004). Por otro lado, el cuestionario incluía una pregunta orientada a medir el nivel de satisfacción que los clientes habían obtenido tras finalizar su experiencia de compra. Finalmente, se añadió una variable dicotómica con objeto de conocer si el folleto publicitario había influido en la compra realizada por el cliente en el establecimiento comercial. La Tabla 2 muestra a continuación las variables introducidas en el estudio, especificando su escala de medida, si se introducen como explicativas, endógenas o de clasificación, y su descripción.

Tabla 2

Síntesis de variables utilizadas

\begin{tabular}{l|l|l|l}
\hline \multicolumn{1}{c|}{$\begin{array}{c}\text { Nombre de la } \\
\text { variable }\end{array}$} & $\begin{array}{c}\text { Escala de } \\
\text { medida }\end{array}$ & $\begin{array}{l}\text { Tipo de variable } \\
\text { en el modelo }\end{array}$ & \multicolumn{1}{c}{ Definición } \\
\hline $\begin{array}{l}\text { Satisfacción } \\
\text { global }\end{array}$ & Métrica* & Endógena & $\begin{array}{l}\text { Satisfacción con la compra realizada en el estable- } \\
\text { cimiento: } \\
\text { Nivel mínimo (1) - Nivel máximo (5) }\end{array}$ \\
\hline $\begin{array}{l}\text { Precio reducido } \\
\text { Promoción } \\
\text { de ventas }\end{array}$ & Métrica & Explicativa & $\begin{array}{l}\text { Percepción del precio (escala de 5 puntos): } \\
\text { Pobre (1) - Excelente (5) }\end{array}$ \\
\hline $\begin{array}{l}\text { Calidad } \\
\text { de la oferta }\end{array}$ & Métrica & Explicativa & $\begin{array}{l}\text { Percepción de la calidad (escala de 5 puntos): } \\
\text { Pobre (1) - Excelente (5) }\end{array}$ \\
\hline $\begin{array}{l}\text { Marcas } \\
\text { comercializadas de la promoción de ventas (escala de 5 } \\
\text { puntos): } \\
\text { Pobre (1) - Excelente (5) }\end{array}$ & Métrica & Explicativa & $\begin{array}{l}\text { Percepción de la marca (escala de 5 puntos): } \\
\text { Pobre (1) - Excelente (5) }\end{array}$ \\
\hline $\begin{array}{l}\text { Cercanía } \\
\text { al hogar }\end{array}$ & Métrica & Explicativa & $\begin{array}{l}\text { Percepción de la cercanía al hogar (escala de 5 } \\
\text { puntos): } \\
\text { Pobre (1) - Excelente (5) }\end{array}$ \\
\hline $\begin{array}{l}\text { Surtido } \\
\text { al cliente }\end{array}$ & Métrica & Explicativa & $\begin{array}{l}\text { Percepción del surtido (escala de 5 puntos): } \\
\text { Pobre (1) - Excelente (5) }\end{array}$ \\
\hline
\end{tabular}




\begin{tabular}{l|l|l|l}
\hline \multicolumn{1}{c|}{$\begin{array}{c}\text { Nombre de la } \\
\text { variable }\end{array}$} & $\begin{array}{c}\text { Escala de } \\
\text { medida }\end{array}$ & $\begin{array}{c}\text { Tipo de variable } \\
\text { en el modelo }\end{array}$ & \multicolumn{1}{c}{ Definición } \\
\hline $\begin{array}{l}\text { Servicios } \\
\text { adicionales }\end{array}$ & Métrica & Explicativa & $\begin{array}{l}\text { Percepción de los servicios adicionales (escala de } \\
5 \text { puntos): } \\
\text { Pobre (1)- Excelente (5) }\end{array}$ \\
\hline $\begin{array}{l}\text { Atmósfera } \\
\text { del } \\
\text { establecimiento }\end{array}$ & Métrica & Explicativa & $\begin{array}{l}\text { Percepción de la atmósfera del establecimiento } \\
\text { (escala de 5 puntos): } \\
\text { Pobre (1)- Excelente (5) }\end{array}$ \\
\hline $\begin{array}{l}\text { Horario de } \\
\text { apertura }\end{array}$ & Métrica & Explicativa & $\begin{array}{l}\text { Percepción del horario de apertura (escala de 5 } \\
\text { puntos): } \\
\text { Pobre (1)- Excelente (5) }\end{array}$ \\
\hline $\begin{array}{l}\text { Influencia del } \\
\text { folleto }\end{array}$ & Categórica & Clasificación & $\begin{array}{l}\text { Influencia del folleto en la compra: } \\
\text { (0) No ejerce influencia } \\
(1) \text { Ejerce influencia }\end{array}$ \\
\hline
\end{tabular}

*Nota: Partiendo de esta variable, y con el fin de analizar la influencia de los factores identificados sobre el nivel más elevado de satisfacción, se elaboró una variable dicotómica a posteriori en la que el valor 1 recogía el máximo nivel de satisfacción del consumidor y el valor 0 , cualquier otro nivel de satisfacción.

\subsection{Planteamiento de hipótesis}

Teniendo en cuenta la relación que la revisión de la literatura establece entre las diferentes variables explicativas relativas a la percepción del consumidor sobre los atributos caracterizadores del establecimiento comercial, y la elevada correlación entre ellas, se aplicó la metodología factorial propuesta por Gómez et al. (2004) con objeto de identificar un conjunto de factores más reducido capaz de explicar la posible variabilidad encontrada en dichas variables. Antes de llevar a cabo tal análisis, se comprobó la fiabilidad de la escala empleada mediante el coeficiente alfa de Cronbach, que alcanzó un valor de 0,713. Al resultar este valor superior a 0,7 , se aseguró la consistencia interna y se confirmó la fiabilidad de la escala de medida (e.g.Nunnally, 1978). Antes de aplicar el método de componentes principales con rotación varimax a las diferentes variables, se analizó también la idoneidad estadística de esta prueba mediante el test de esfericidad de Bartlett y la medida de Kaiser-Meyer-Olkin. Como el test de esfericidad de Barlett tomó un valor alto nos permitió rechazar la hipótesis nula de no correlación significativa con un nivel de significación del 0,000, se corroboró la conveniencia de aplicar el análisis de componentes principales a las variables explicativas consideradas. Adicionalmente, la medida de adecuación de KaiserMeyer-Olkin (el comúnmente referido KMO) adquirió un valor de 0,760, valor superior al límite de 0,5 establecido para este índice (George y Mallery, 1995).

$\mathrm{El}$ análisis factorial de componentes principales permitió obtener tres factores que explicaban el 56,4\% de la variación en las variables consideradas (ver Tabla 3). En concreto, el factor denominado "servicios y conveniencia" (SC), recoge las variables de variedad del surtido, proximidad al hogar, atención al cliente, servicios adicionales y atmósfera del establecimiento. El factor "calidad e imagen" (CI) incluye las variables de calidad percibida de los productos ofrecidos y marcas comercializadas. Y el factor "valor económico" (VE) agrupa las variables precio y promociones de ventas. 
Tabla 3

Factores del establecimiento comercial subyacentes a los atributos considerados

\begin{tabular}{l|l|c}
\hline \multicolumn{1}{c|}{ Factor } & \multicolumn{1}{|c}{ Atributos que lo componen } & Puntuación \\
\hline Servicios y conveniencia $(S C)$ & Amplitud del surtido & 0,540 \\
& Proximidad al hogar & 0,564 \\
& Atención al cliente & 0,604 \\
& Servicios adicionales & 0,661 \\
& Atmósfera del establecimiento & 0,823 \\
& Horario de apertura & 0,742 \\
\hline Calidad e imagen $(C I)$ & Calidad & 0,660 \\
& Marcas comercializadas & 0,772 \\
\hline Valor económico de la compra $(\mathrm{VE})$ & Precio & 0,849 \\
& Promoción & 0,837 \\
\hline
\end{tabular}

Estos resultados no resultan muy diferentes a los obtenidos por otros trabajos realizados con datos procedentes de otros países. Por ejemplo, Gómez et al. (2004), tras analizar una muestra de consumidores norteamericanos, concluyen en señalar la presencia de estos tres mismos factores como determinantes de la capacidad diferenciadora del establecimiento comercial, haciendo especial énfasis en el impacto que se deriva de la percepción del cliente sobre su satisfacción global. En la misma línea, el trabajo de Binninger (2008), mediante el estudio de una muestra de consumidores franceses, no sólo detecta el efecto directo que la calidad percibida del producto ejerce sobre la satisfacción del cliente, sino que además indica la existencia de un efecto indirecto relacionado con el valor que el cliente atribuye a la marca del distribuidor. En los trabajos de Zeithaml (1988), McDougall y Levesque (2002) y Grace y O’Cass (2005) también se observa una relación entre la dimensión económica de la compra y la satisfacción que el sujeto experimenta con la misma. En España, el trabajo realizado por Bigné y Andreu (2004) coincide en señalar cómo determinados servicios ofrecidos al cliente (e.g., atención al cliente, facilidad de acceso, etc.) ejercen un gran efecto positivo sobre la satisfacción global del cliente en centros comerciales, resultando este efecto menor en establecimientos situados en el centro de las ciudades.

Partiendo de estos trabajos -entre otros-, se puede afirmar la existencia de una línea de investigación que reconoce el impacto que los factores caracterizadores del establecimiento comercial son susceptibles de ejercer sobre la satisfacción global del consumidor. No obstante, tal y cómo se apuntaba al inicio de este trabajo, el objetivo que se plantea en este trabajo tiene que ver con el nivel máximo de satisfacción del consumidor, y no con su nivel de satisfacción global. Y ello debido a que con frecuencia, las actividades de algunos de estos distribuidores en el sector están condicionadas sobremanera por la consideración de servir el máximo nivel de calidad percibida como objetivo prioritario (Spreng y Mackoy, 1996). 
Así pues, el interés principal de este trabajo se centra en el estudio de la influencia que ciertos factores caracterizadores del establecimiento comercial son susceptibles de ejercer sobre el nivel de satisfacción máxima del consumidor. Teniendo en cuenta que este nivel de satisfacción podrá estar condicionado por la influencia de aspectos tales como los folletos publicitarios -dado su impacto sobre los procesos de búsqueda y evaluación de alternativas por parte del consumidor-, el objetivo de investigación permite llevar a cabo el planteamiento de las siguientes hipótesis centradas en la influencia de los anteriores factores sobre el nivel más elevado de satisfacción, e incorporando la influencia del folleto publicitario:

H1. La percepción de los servicios y de la conveniencia que el establecimiento comercial ofrece al cliente influye de manera positiva y significativa en su nivel máximo de satisfacción.

H2. La percepción de la imagen de calidad que el cliente obtiene de su experiencia de compra en el establecimiento comercial influye de manera positiva y significativa en su nivel de satisfacción máximo.

H3. La percepción sobre el valor económico de la compra realizada por el cliente en el establecimiento influye de manera positiva y significativa en su nivel de satisfacción máximo.

Por tanto, el contrate de estas hipótesis se efectuará considerando la influencia del folleto publicitario en la compra. En el estudio empírico que se describe en la sección siguiente se abordará el análisis de la influencia que los factores identificados ejercen sobre el nivel de satisfacción máxima del consumidor analizando dos submuestras de consumidores que han reconocido que su compra: (1) ha estado influida por el folleto publicitario y (2) no ha estado influida por el folleto publicitario.

\section{OBTENCIÓN Y ANÁLISIS DE RESULTADOS}

Con el fin de determinar el impacto que los factores previamente identificados ejercen sobre el nivel más elevado de satisfacción que el consumidor obtiene, teniendo además en cuenta la influencia del folleto publicitario en la compra, se ha realizado un análisis de regresión logístico binario. Para que este análisis fuera viable, se ha transformado la variable de satisfacción global introducida inicialmente en la encuesta en una variable dicotómica orientada a recoger exclusivamente el nivel máximo de satisfacción. Esta nueva variable ha sido codificada de tal modo que el valor 1 recoge el nivel máximo de satisfacción del consumidor y el valor 0 recoge cualquier otro nivel de satisfacción. En la Tabla 4 se describen los estadísticos descriptivos de los factores, diferenciando entre distintas submuestras de consumidores en función de si reconocen que su compra ha estado influida o no por el folleto publicitario. 
Tabla 4

\section{Estadísticos descriptivos de los factores}

\begin{tabular}{|c|c|c|c|}
\hline Variables explicativas & $\begin{array}{c}\text { El folleto publicitario } \\
\text { influye } \\
(\mathrm{N}=230)\end{array}$ & $\begin{array}{c}\text { El folleto publicitario } \\
\text { no influye } \\
(\mathrm{N}=192) \\
\end{array}$ & Significación ${ }^{(a)}$ \\
\hline Media SC & $-0,707$ & $-0,415$ & \\
\hline (desviación típica) & 1,081 & 1,117 & \\
\hline$($ varianza $)$ & 1,170 & 1,250 & \\
\hline (mediana) & $-0,866$ & $-0,447$ & $*$ \\
\hline Media CI & $-0,366$ & $-0,215$ & \\
\hline (desviación típica) & 1,067 & 0,970 & \\
\hline (varianza) & 1,139 & 0,942 & \\
\hline (mediana) & $-0,195$ & $-0,257$ & \\
\hline Media EV & 0,236 & 0,211 & \\
\hline (desviación típica) & 0,857 & 0,878 & \\
\hline (varianza) & 0,735 & 0,771 & \\
\hline (mediana) & 0,443 & 0,161 & $* * *$ \\
\hline
\end{tabular}

Esta tabla muestra cómo los valores medios más elevados de los factores de servicios y conveniencia y calidad e imagen, se detectan en aquellas compras en las que el folleto publicitario no influye. Por el contrario, las medias más altas del factor relativo al valor económico de la compra se observan en aquella muestra de consumidores en las que el folleto publicitario sí influye. A su vez, los factores de servicios y conveniencia y valor económico de la compra poseen los valores más elevados de la varianza en las muestras de consumidores en las que el folleto no ejerce ningún efecto. Sin embargo, cuando el folleto publicitario sí ejerce influencia en el acto de compra, la varianza más elevada se detecta en el factor de calidad e imagen.

La Tabla 5 muestra el ratio de probabilidades de que el consumidor quede totalmente satisfecho con la compra realizada en el establecimiento, que varía entre 3,358 -para los consumidores cuya compra está influida por el folleto publicitario- y 2,604 -para los consumidores cuya compra no está influida por el folleto publicitario-.

Tabla 5

\section{Tabla de clasificación}

\begin{tabular}{l|c|c}
\hline & El folleto influye $(\mathrm{N}=231)$ & El folleto no influye $(\mathrm{N}=191)$ \\
\hline Tasa base de satisfacción máxima & $178 / 231=0,770$ & $138 / 191=0,722$ \\
\hline Probabilidad observada & $178 / 53=3,358$ & $138 / 53=2,604$ \\
\hline
\end{tabular}


Asimismo, la Tabla 6 presenta los valores de los pseudos $\mathrm{R}^{2}$ de Cox y Snell, Nagelkerke y el -2 log de la verosimilitud obtenidos para las diferentes submuestras de compradores identificadas en función de la influencia del folleto publicitario.

Tabla 6

\section{Resumen de los modelos}

\begin{tabular}{l|c|c|c}
\hline $\begin{array}{c}\text { Influencia del folleto } \\
\text { publicitario en la compra }\end{array}$ & $-2 \log$ de la verosimilitud & $\begin{array}{c}\text { R cuadrado de Cox y } \\
\text { Snell }\end{array}$ & $\begin{array}{c}\text { R cuadrado de } \\
\text { Nagelkerke }\end{array}$ \\
\hline $\mathrm{Si}$ & 216,411 & 0,131 & 0,199 \\
\hline $\mathrm{No}$ & 205,538 & 0,100 & 0,144 \\
\hline
\end{tabular}

Estos valores son razonables en todas las submuestras consideradas. La mayor capacidad explicativa se detecta en aquella muestra de consumidores en la que el folleto publicitario influye, donde el modelo es capaz de explicar entre un $13,1 \%$ y un $19,9 \%$ en la variabilidad de la probabilidad de la variable de satisfacción máxima introducida. Por el contrario, la menor capacidad explicativa recae en el modelo realizado en la submuestra de compradores en las que el folleto publicitario no influye.

Tabla 7

Relación de las variables explicativas y el nivel de satisfacción más elevado

\begin{tabular}{l|c|c}
\hline Parámetros estimados de los factores & El folleto publicitario influye & El folleto publicitario no influye \\
\hline Constante & $\mathbf{1 , 4 0 9 * * *}$ & $\mathbf{0 , 9 4 2} * * *$ \\
\hline SC & $\mathbf{0 , 7 7 1} * * *$ & $\mathbf{, 6 2 5} * * *$ \\
\hline CI & $\mathbf{0 , 4 0 9 * * *}$ & 0,167 \\
\hline VE & 0,295 & $\mathbf{- 0 , 2 9 3} *$ \\
\hline $\mathrm{p}<0,10 ; * * \mathrm{p}<0,05 ; * * * \mathrm{p}<0,01$ & \multicolumn{2}{|l}{} \\
\hline
\end{tabular}

El análisis de la Tabla anterior pone de manifiesto cómo para la muestra de consumidores en la que el folleto publicitario influye, los factores que contribuyen de manera positiva y significativa a incrementar la probabilidad de que el consumidor obtenga un nivel máximo de satisfacción con su compra son el de servicios y conveniencia, seguido por el de imagen y calidad. Sin embargo, en la muestra de consumidores que no reconoce sentirse influidos por el folleto publicitario a la hora de realizar su compra, el único factor que contribuye positiva y significativamente a aumentar la probabilidad de que el consumidor obtenga un nivel de satisfacción máximo con su compra es el de servicios y conveniencia. Y es que para estos consumidores, la percepción del factor de valor económico de la compra influye de forma negativa y significativa en el nivel de satisfacción máximo del consumidor. 
Estos resultados evidencian cómo el factor relativo a la percepción de los servicios y la conveniencia que el establecimiento comercial ofrece al cliente ejerce una influencia positiva y significativa en el nivel máximo de satisfacción con las compras realizadas por todos los consumidores, tanto si están influidos como si no están influidos por el folleto publicitario. Por tanto, la primera de las hipótesis propuestas relativa a la influencia positiva y significativa de los servicios y conveniencia ofrecidos por el detallista en relación con el nivel máximo de satisfacción del consumidor, se puede aceptar tanto para la muestra de consumidores en la que el folleto publicitario influye como en aquella muestra de consumidores en las que el folleto publicitario no influye. Por otra parte, el factor relativo a la percepción de la calidad e imagen que el distribuidor detallista ofrece sólo ejerce una influencia significativa sobre la muestra de consumidores en las que se reconoce la influencia del folleto publicitario, por lo que únicamente es posible aceptar la segunda hipótesis propuesta en esta submuestra. Por último, el factor referido al valor económico de la compra no influye de forma positiva y significativa en el nivel de satisfacción máxima de ninguna de las compras, por lo que no es posible aceptar en ningún caso la tercera de las hipótesis propuestas. En la comprensión de este resultado, es preciso tener en cuenta el contexto temporal en el que se recogió la información: la crisis económica no hacía más que comenzar, y quizás muchos consumidores no eran conscientes de la misma.

El Cuadro 1 muestra a continuación el resumen del contraste de hipótesis realizado en función de la submuestras de consumidores considerada.

\section{Cuadro 1}

Factores que influyen sobre la máxima satisfacción del consumidor - Diferencias en función del formato de establecimiento

\begin{tabular}{l|c|c}
\hline \multicolumn{1}{c|}{ Muestra de turistas considerada } & Hipótesis que se aceptan & Hipótesis que no se aceptan \\
\hline Influencia del folleto publicitario & $\mathrm{H} 1 ; \mathrm{H} 2$ & $\mathrm{H} 3$ \\
\hline No influencia del folleto publicitario & $\mathrm{H} 1$ & $\mathrm{H} 2 ; \mathrm{H} 3$ \\
\hline
\end{tabular}

Estos hallazgos ponen de manifiesto la elevada importancia que alcanza los atributos integrados en el factor de servicios y conveniencia por parte del consumidor, independientemente de que el folleto publicitario ejerza influencia o no sobre la compra. También se ha evidenciado que el factor de calidad e imagen influye positivamente en las compras de aquellos consumidores en los que influye el folleto publicitario. Por último, los atributos de precio y promoción de ventas incluidos en el factor de valor económico de la compra, no sólo no ejercen influencia sobre el nivel máximo de satisfacción del consumidor, sino que cuando influyen en aquellos consumidores en los que no influye el folleto publicitario, lo hacen de forma negativa.

Por tanto, los responsables comerciales de la gestión comercial de estos establecimientos, a la hora de llevar a cabo el diseño y la difusión de este tipo de folletos, no sólo deberían utilizarlos cómo refuerzo a los descuentos de precio promocionales, sino que resultaría útil considerarlos como un mecanismo para comunicar propuestas de valor a los consumidores 
relacionadas con los servicios, la conveniencia, la calidad e imagen. Propuestas de valor que deberían enfatizar y hacer llegar a través de otros medios a aquellos consumidores en cuyas compras no afectan los folletos publicitarios.

\section{CONCLUSIONES Y RECOMENDACIONES PARA LA GESTIÓN}

Dada la relevancia y connotaciones estratégicas del sector de la distribución minorista de productos de gran consumo, los operadores minoristas que actúan en el mismo deben buscar las vías más adecuadas para tratar de mantener y, en la medida de lo posible, superar, su posición competitiva. De este modo, resulta fundamental que las empresas minoristas centren sus esfuerzos en aquellas capacidades internas más adecuadas sobre las que construir sus estrategias competitivas, además de desarrollar las habilidades necesarias para identificar el modo en el que los clientes perciben tales capacidades. No en vano, la práctica real ha demostrado cómo gran parte de la capacidad competitiva de estos comerciantes esta condicionada por el conocimiento que tengan de sus clientes.

Por este motivo, conocer y gestionar correctamente la información existente sobre los consumidores es clave para poder: (1) conocer cuáles son los elementos del establecimiento comercial que son valorados por los clientes; (2) determinar cuáles son los factores subyacentes a dichos elementos y (3) identificar cuáles de éstos presentan una mayor influencia sobre la satisfacción del consumidor. Y todo ello, con el objetivo de diseñar y poner en práctica estrategias comerciales que los potencien a fin de satisfacer adecuadamente las necesidades de los clientes a la vez de lograr un posicionamiento diferencial en los mercados.

Con el ánimo de identificar los factores que los consumidores más valoran en relación con los atributos diferenciadores de los establecimientos minoristas de alimentación, así como su relación con los niveles más elevados de satisfacción del consumidor, se ha efectuado una investigación empírica en la que primeramente se han considerado los atributos clave del establecimiento comercial que la literatura relevante había identificado como fundamentales para que los comerciantes en el sector logren diferenciarse de sus competidores. Este planteamiento inicial ha permitido detectar los principales factores que subyacen a dichos aspectos, determinando posteriormente cuáles de éstos ejercen una mayor influencia sobre el nivel de satisfacción más elevado del consumidor. Con este objetivo, se ha procedido a analizar la información procedente de una muestra de 422 compradores que había sido recogida en establecimientos de alimentación que operan en España en régimen de libreservicio bajo diversos formatos: hipermecados, supermercados, tiendas de conveniencia y establecimientos de descuento. El análisis se ha realizado teniendo en cuenta una variable clave caracterizadora del comportamiento de los consumidores como es la influencia del folleto publicitario, actividad que en la coyuntura actual ha cobrado especial importancia.

Los hallazgos encontrados han puesto de manifiesto cómo la percepción de los servicios y la conveniencia que el comerciante minorista de productos de gran consumo ofrece al consumidor ejerce una influencia positiva y significativa sobre el nivel máximo de satisfacción del consumidor con la compra realizada, y ello con independencia de que la compra esté influida o no por el folleto publicitario. Ahora bien, la percepción de la imagen y calidad del distribuidor sólo ejerce una influencia significativa y positiva sobre las compras 
de aquellos sujetos que sí tienen en cuenta la información recogida en el folleto publicitario a la hora de realizar su compra. Sin embargo, la percepción del valor económico de la compra no influye sobre el nivel más elevado de satisfacción del consumidor en aquellas compras en las que el folleto publicitario no ejerce influencia, realizando por otro lado una influencia negativa sobre las compras de aquellos consumidores que no tienen en cuenta el folleto publicitario.

De este modo, en general destaca la influencia tan reducida que ejerce el factor de valor económico de la compra y los atributos que lo componen (precios y promociones), que incluso influye de forma negativa sobre la satisfacción máxima de aquellos consumidores que no consideran en absoluto la información recogida en el folleto publicitario (debido quizás al propio contexto económico durante el que se recogieron los datos). También, cabe señalar que el factor de calidad e imagen sólo influye -y en menor medida en comparación a la influencia del factor de servicios y conveniencia- sobre la satisfacción máxima de aquellos consumidores que tienen en cuenta los folletos promocionales a la hora de realizar sus compras.

Este estudio ha posibilitado la obtención de interesantes conclusiones y recomendaciones que resultan útiles para la gestión de establecimientos minoristas de alimentación que operan en régimen de libreservicio. En concreto, ha permitido observar tanto los atributos del establecimiento comercial como los factores subyacentes a los mismos que los consumidores más valoran y que contribuyen a los niveles de satisfacción más elevados. Esto sin duda facilita a los responsables de acometer la gestión comercial en estos establecimientos de disponer de elementos válidos de valoración sobre los puntos fuertes/ débiles que se han de vigilar con el objetivo de mantener y mejorar, en la medida de lo posible, una determinada posición competitiva. Adicionalmente, también hace posible el diseño e implementación de estrategias de marketing que hagan factible la gestión integral y eficiente de aspectos relativos a los servicios y la conveniencia ofrecidos, la amplitud y profundidad de la oferta de productos y marcas, la imagen y calidad del establecimiento o el valor monetario de la compra.

Este estudio también ha proporcionado elementos fiables que los operadores en el sector pueden tener en cuenta a la hora de proceder a diseñar adecuadamente estos folletos promocionales. En efecto, este trabajo ha puesto de manifiesto cómo los responsables de llevar a cabo la gestión comercial, a la hora de diseñar este tipo de folletos, deberían considerarlos no sólo como elementos de apoyo a los descuentos de precio y otras promociones, sino también como instrumentos que permitan comunicar propuestas de valor relacionadas con los servicios, conveniencia, calidad e imagen que el establecimiento comercial ofrece.

No obstante, el presente estudio no carece de limitaciones, entre las que cabe mencionar la falta de generalización de los resultados más allá de los propios consumidores que componen la muestra, puesto que se ha utilizado un procedimiento de muestreo no probabilístico. O también, las propias limitaciones derivadas de aquellos atributos del establecimiento comercial que no han sido considerados, como por ejemplo, atributos a nivel corporativo.

Como líneas futuras de investigación, se propone el estudio de cómo los factores identificados pueden influir en el grado de selección de determinadas categorías de productos, considerando otros aspectos del consumidor (e.g., frecuencia de compra, importancia de la compra por impulso, la respuesta a otras actividades promocionales) o del propio comer- 
ciante (e.g., formato del establecimiento). Asimismo, también se considera de interés para futuros trabajos de investigación la incorporación de atributos del establecimiento comercial relacionados con aspectos de tipo institucional o corporativo.

\section{REFERENCIAS BIBLIOGRÁFICAS}

ANDERSON, J. L.; JOLLY, O. L. D Y FAIRHURST, A. E. (2007). “Customer relationship management in retailing: A content analysis of retail trade journals", Journal of Retailing and Consumer Services, Vol. 14, pgs. 394-399.

BELLENGER, D. Y KORGAONKAR, P. (1980). "Profiling the recreational shopper". Journal of Retailing, 56, pgs. 77-92.

BELLENGER, D.; ROBERTSON, D. Y GREENBURG, B. (1977), "Shopping center patronage motives", Journal of Retailing, Vol..2, pgs.29-38.

BERNÉ, C. (2006). "Análisis de la demanda de servicios de distribución minorista", Información Comercial Española (ICE), 828, pgs.83-98

BERNÉ, C. Y MARTÍNEZ, N. (2007). "Determinantes del comportamiento variado del consumidor en el escenario de compra”, Documentos de trabajo de la Universidad de Zaragoza (2007-01).

BERNHARDT, K.; DONTHU, N. Y KENNETT, P. (2000). "A longitudinal analysis of satisfaction and profitability”, Journal of Business Research, Vol. 47, pgs. 161-171.

BERRY, L. L.; SEIDERS, K. Y GREWAL, D. (2002). “Understanding Service Convenience”, Journal of Marketing, Vol. 66, $\mathrm{n}^{\circ}$ 3, pgs. 1-17.

BETANCOURT, R. R., CORTIÑAS, M.; ELORZ, M. Y MUGICA, J.M. (2007). "The demand for and the supgsly of distribution services: A basis for the analysis of customer satisfaction in retailing", Quantitative Markets and Economy, Vol. 5, pgs. 293-312.

BICE (2006). "La distribución comercial en la Unión Europea", Boletín de Información Comercial Española, Vol. 2877 (del 1 al 14 de mayo), pgs. 185-206.

BIGNÉ E. Y ANDREU, L. (2004). "Emociones, satisfacción y lealtad del consumidor en entornos comerciales: Un análisis comparativo entre centro comercial y centro urbano", Distribución y Consumo, Vol. 77, pgs. 77-87.

BINNINGER, A.S. (2008). "Exploring the relationship between retail brands and consumer store loyalty", International Journal of Retail \& Distribution Management, Vol. 36, nº 2. pgs, 94-110.

BLATTBERG, R. G.; BRIESCH, R. Y FOX, E. (1995), "How Promotions Works", Marketing Science, Vol. 14, $\mathrm{n}^{\circ}$ 3, G122-G132.

BRIDSON, K.; EVANS, J. Y HICKMAN, M. (2008). "Assessing the relationship between loyalty program attributes, store satisfaction and store loyalty", Journal of Retailing and Consumer Services, Vol. 15, pgs. 364-374.

BUIL, I. Y MONTANER, T. (2007): "Influencia de los hábitos de compra en la respuesta del consumidor ante las promociones”, Distribución y Consumo, Vol. mayo-junio, pgs. 63-69.

BURTON, S.; LICHTENSTEIN, D. Y NETEMEYER, R. (1999), "Exposure to Sales Flyers and Increased Purchases in Retail Supermarkets", Journal of Advertising Research, septiembreoctubre, pgs. 7-14.

CORSTJENS, M. Y LAL, R. (2000). "Building stores royalty through store brands", Journal of Marketing Research, Vol. 37, no. 3, pgs. 281-291.

CRAIG, C.S., GHOSH, A. Y MCLAFFERTY, S. (1984). "Models of the retail location process: a review", Journal of Retailing, Vol. 60, pgs. 5-36.

DARDEN, W. R. Y SCHWINGHAMMER, J. K. L. (1985). "The Influence of Social Characteristics on Perceived Quality in Patronage Choice Behavior", en J. Jacoby and J. Olson (eds.), Perceived quality: Institute of retail management, New York University. Lexington Books. 
DAVIS-SRAMEK, B.; STANK, T. P. Y MENTZER, J. T. (2008). "Creating Consumer Durable Retailer Customer Loyalty through Order Fulfillment ServiceOperations”, Journal of Operations Management, Vol. 26, $\mathrm{n}^{\circ} 6$.

DELLAERT, B.; ARENTZE, T.; BIERLAIRE, M.; BORGERS, A. Y TIMMERMANS, H. (1998). "Investigating consumers'tendency to combine multiple shopgsing purposes and destination", Journal of Marketing Research, Vol. 35, n 2, pgs. 177-188.

DODDS, W. B. (1995). "Market cues affect on consumers' product evaluation", Journal of Marketing Theory and Practice, Vol. 3, $\mathrm{n}^{\circ} 2$, pgs. 50-63.

DONOVAN, R.; ROSSISTER, J.; MARCOOLYN Y NESDALE, A. (1994). "Store atmosphere and purchase behavior", Journal of Retailing, Vol. 70, n 3, pgs. 283-94.

DUNNE, P. Y KAHN, R. (1997). "Retailing in the USA: An Interpretation of Current Trends", International Journal of Retail and Distribution Management, Vol. 25, $\mathrm{n}^{\circ}$ 8,pgs. 275-281.

ELDIARIOMONTANES.ES (2009): "Las cajeras, “carne de cañón”, disponible en http://www.eldiariomontanes.es/20090320/cantabria/otras-noticias/cajeras-carne-canon-20090320.html.

EL ECONOMISTA.ES (2008): "Se confirma el frenazo: España sólo creció el 0,1\% en el segundo trimestre", disponible en http://www.eleconomista.es/economia/noticias/702662/08/08/Se-confirma-el-frenazo-Espana-solo-crecio-el-01-en-el-segundo-trimestre.html

FINN, A. Y LOUVIERE, J. (1996). "Shopping center image, consideration, and choice: anchor store contribution", Journal of Business Research, Vol. 35, n' marzo, pgs. 241-251.

FRASQUET, M. Y MOLLÁ, A. (2000): "Modelización de la selección de centro comercial a partir de las dimensiones de valor percibido", Revista Española de Investigación de Marketing ESIC, VOL. 4, PGS. 81-108.

GANESH, J.; REYNOLDS, K.E, Y LUCKETT, M.G. (2007). "Retail patronage behaviour and shopgser typologies: A replication and extension usin a multi-format, multi-method apgsroach", Journal of the Academy of Marketing Science, Vol. 35, pgs. 369-381.

GÁZQUEZ, J. C. Y SÁNCHEZ, M. (2006): "Sobre la eficacia de la promoción de productos de gran consumo: Folletos versus descuentos en precios", Distribución y Consumo, Vol. marzo-abril, pgs. 5-13.

GENTRY, J. W. Y BURNS, A. (1977). "How important are evaluative criteria in shopgsing center patronage", Journal of Retailing, Vol. 53, pgs. 73-86.

GEORGE, D. Y MALLERY, P. (1995). SPSS/PC+ Step by step. A simple guide and reference.

GÓMEZ, M. Y RUBIO, N. (2006). "Gestión minorista de las marcas de distribuidor: Producto y merchandising", Información Comercial Española, Vol. 828, pgs. 157-174.

GÓMEZ, M; MCLAUGHLING, E. Y WITTINK, D. R. (2004). "Customer satisfaction and retail sales performance: An empirical investigation”, Journal of Retailing, Vol. 80, pgs. 265-278.

GONZÁLEZ, O. (2005): "Papel de la localización en la selección de establecimiento minorista: un enfoque tridimensional", Distribución y Consumo, Vol. marzo-abril, pgs.100-107.

GRACE, D. Y O'CASS, A. (2005). "An examination of the antecedents of patronage intentions across different retail store formats", Journal of Retailing and Consumer Services, Vol. 12, $\mathrm{n}^{\circ}$ 4, pgs. 227-243.

HANSEN, R. Y DEUTSCHER, T. (1997/1978). "An empirical investigation of attribute importance in retail store selection", Journal of Retailing, Vol. 53.

HENNIG-THURAU, T. Y KLEE, A. (1997). "The Impact of Customer Satisfaction and Relationship Quality on Customer Retention: A Critical Reassessment and Model Development”, Psychology \& Marketing, Vol. 14, 8, pgs. 737-764.

HOWARD, E. (1997). "The management shopgsing centres: conflict or collaboration?", The International Review of Retail, Distribution and Consumer Research, Vol. 7, n 3, pgs.263-285.

INFOBAE.COM (2008). "Advierten que España sufrirá una gran crisis económica”, disponible en http://www.infobae.com/economia/403685-100884-0-Advierten-que-España-sufrirá-una-grancrisis-económica. 
JOHNSON, M.D., GUSTAFSSON. Y ANDREASSEN, T.W. (2001). "The evolution and future of national customer satisfaction index models", Journal of Economic Psychology, Vol. 22, 2, pgs.217-245.

KOTLER, P. (1973). "Atmospherics as a marketing tool”, Journal of retailing, Vol. 49, $\mathrm{n}^{\circ}$ invierno, pgs. 48-64.

LA CAIXA (2008). "Anuario Económico”, disponible en http://www.anuarieco.lacaixa.comunicacions.com.

LARSON, R. B. (2004). "New Market Groupings Based on Food Consumption Patterns", Agribusiness, Vol. 20 (4), pgs. 417-432.

LEVY, M. Y WEITZ, B.A. (1995). Retailing Management, Irwin, Chicago.

MARTÍNEZ RUIZ, M. P. (2005). La influencia de las características del descuento de precio promocional y de la marca en el incremento de las ventas: aplicación de la metodología SVM con datos de escáner minorista, Alicante: Biblioteca Virtual Miguel de Cervantes, [disponible en http://cervantesvirtual.es].

MARTÍNEZ RUIZ, M. P. Y MOLLÁ DESCALS, A. (2007). "Implicaciones de la estrategia de precios Hi-Lo en la industria de productos de gran consumo", Universia Business Review, Vol. $15, \mathrm{n}^{\circ}$ 3er trimestre, pgs. 10-25.

MCCOLLOUGH, M., BERRY, L. Y YADAV, M. (2000). "An Empirical Investigation of Customer Satisfaction after Service Failure and Recovery", Journal of Service Research, Vol. noviembre, pgs. 121-137.

MCDOUGALL, G. H. Y LEVESQUE, T. J. (1994). "A revised view of service quality dimensions: An empirical investigation", Journal of Professional Services Marketing, Vol. 11, n 1, pgs. 189-209.

MOLERO, V. Y PUELLES, M. (2009). "El marketing en las rebajas: Impacto económico y efecto en los consumidores", Distribución y Consumo, Vol. enero-febrero, pgs. 102-112.

MOLINA, A.; MARTÍN-CONSUEGRA, D. Y ESTEBAN, A. (2006). "Marketing de relaciones en la distribución comercial", Distribución Comercial, Vol. noviembre-diciembre, pgs. 127-136.

MOORE, M., Y CARPENTER, J. (2006). "The effect of price as marketplace cue on retail patronage", Journal of Product and Brand Management, Vol. 15, nº. 4. pgs. 265-271.

NUNNALLY, J. C (1978). Psychometric Theory, Vol. 10, septiembre, pgs. 197-208.

PAN Y. Y ZINKHAN, G. (2006). "Determinants of retail patronage: A meta-analytical perspective", Journal of Retailing, Vol. 82, pgs. 229-243.

PARASURAMAN, A.; ZEITHAML, V. Y BERRY, L. (1988). "Servqual: A multiple-Item Scale for Measuring Consumer Perceptions of Service Quality", Journal of Retailing, Vol. 64, $\mathrm{n}^{\circ}$ primavera, pgs. 12-40.

PIRON, F. (2001). "Effects of service and communication initiatives on retail grocery consumers' loyalty", Singapore Management Review, Vol. 23, pgs. 45-61.

QUELCH, J. (2008). "How to market in a recession", Marketing KnowHow, 24 September 2008, disponible en la Web: http://conversationstarter.hbsp.com/2008/10/a_financial_crisis_fifty_ years.html

RAO, A. R. Y MONROE, K. B. (1988). "The Moderating Effect of Prior Knowledge on Cue Utilization in Product Evaluations", Journal of Consumer Research, Vol. 15, $\mathrm{n}^{\mathrm{o}}$ septiembre, pgs. 253-264.

REILLY, W. J. (1931). The Law of Gravitation, Knickerbocker Press, Nueva York.

RUBENSTEIN, C., Y SHAVER, P. R. (1980). "Loneliness in two northeastern cities". en J. Hartog, J. R. Audy, \& Y. A. Cohen (Eds.), The anatomy of loneliness (pgs. 319-337). New York: International Universities Press.

RUST, R. Y ZAHORIK, A.J. (1993). "Customer Satisfaction, Customer Retention and Market Share", Journal of Retailing, Vol.69 (verano), pgs. 193-215. 
SAINZ DE VICUÑA, J. M. (2000). La distribución comercial: Opciones estratégicas, Esic, $2^{\text {a }}$ edición, Madrid.

SEIDERS, K.; VOSS, G. B.; GREWAL, D. Y GODFREY, A. L. (2005). "Do Satisfied Customers Really Buy More? Examining Moderating Influences in a Retailing Context," Journal of Marketing, Vol. 69, $\mathrm{n}^{\circ}$ Octubre, pgs. 26-43.

SPARKS, L. (1995). "Customer service in retailing", en Akehrst G. and A. Nicholas A. (ed), London.

SIVADAS, E. Y BAKER-PREWITT, J. L. (2000). "An examination of the relationship between service quality, Customer Satisfaction and Store Loyalty", International Journal of Retail and Distribution Management, Vol. 28, $\mathrm{n}^{\circ}$ 2, pgs. 73-82.

SPILLER, A.; BOLTEN, J. Y KENNERKNECHT, R. (2006). "Customer satisfaction and loyalty as success factors in organic food retailing", trabajo presentado en el 16th Annual World Forum and Symposium "Agribusiness, Food, Health, and Nutrition”, IAMA Conference, 10-13 de junio, Buenos Aires, Argentina.

SPRENG, R. A. Y MACKOY, R. D. (1996). "An empirical examination of a model of perceived service quality and satisfaction". Journal of Retailing, Vol. 72, 2, pgs. 201-214.

SZYMANSKI, D. Y HENARD, D. H. (2001). "Customer satisfaction: A meta-analysis of the empirical evidence", Academy of Marketing Science Journal, Vol. 29, no 1, pgs. 16-35.

TAUBER, E. M. (1972). "Discovering new product opgsortunities with problem inventory analysis", Journal of Marketing, Vol. 39, pgs. 67-70.

TELLIS, G. J. Y GAETH, G. G. (1990). "Best-Value, Price-Seeking and Price Aversion: The Impact of Information and Learning on Consumer Choices", Journal of Marketing, Vol. 54, $\mathrm{n}^{\circ}$ Abril, pgs. 34-45.

THEODORIDIS, P. K. Y CHATZIPANAGIOTOU, K. C. (2009). "Store image attributes and customer satisfaction across different customer profiles within the supermarket sector in Greece", European Journal of Marketing, Vol. 43 no. 5/6, pgs. 708-734.

UNIÓN EUROPEA (2008). European Comission: Trade, disponible en http://ec.europa.eu/trade/

YU, Y. Y. Y DEAN, A. (2001). "The contribution of emotional satisfaction to consumer loyalty", International Journal of Service Industry Management, Vol. 12, pgs. 234-250. 


\section{Gipuzkoaren kanpo harremanak, esku onetan.}

Las relaciones externas de Gipuzkoa, en buenas manos.

Les relations externes de Gipuzkoa, en bonnes mains.

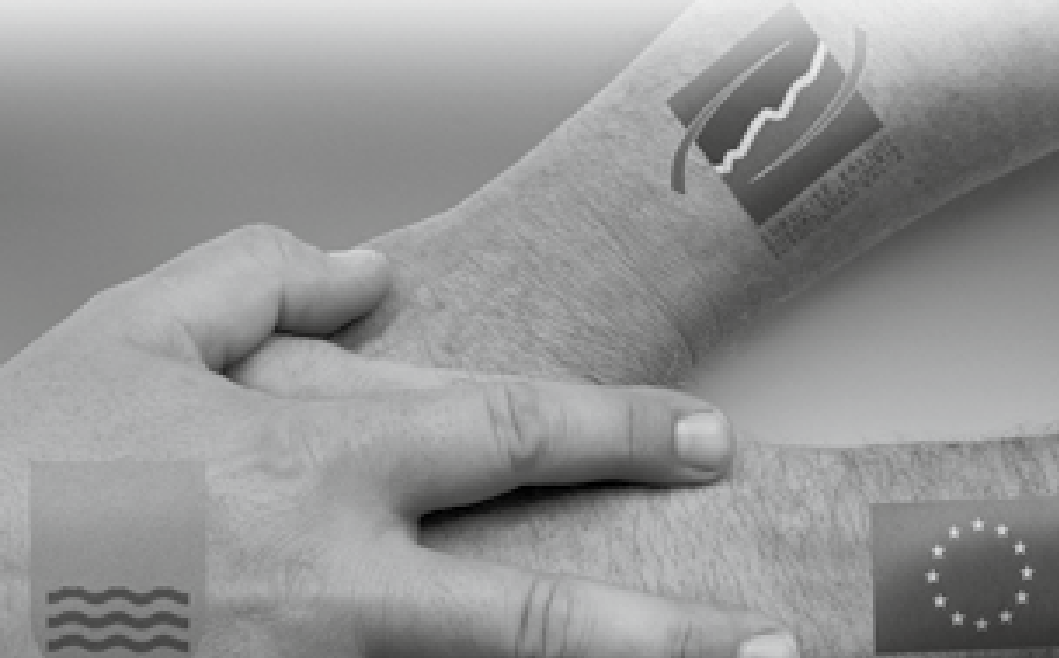

